\title{
A Novel Method for Acquiring Engineering-Oriented Operational Empirical Knowledge
}

\author{
Lijun Liu, Zuhua Jiang, Bo Song, Hongyuan Zhu, and Xinyu Li \\ Department of Industrial Engineering, Shanghai Jiao Tong University, 800 Dongchuan Road, Minhang District, \\ Shanghai 200240, China
}

Correspondence should be addressed to Zuhua Jiang; zhjiang@sjtu.edu.cn

Received 26 September 2015; Revised 26 January 2016; Accepted 16 February 2016

Academic Editor: Vincent Hilaire

Copyright (C) 2016 Lijun Liu et al. This is an open access article distributed under the Creative Commons Attribution License, which permits unrestricted use, distribution, and reproduction in any medium, provided the original work is properly cited.

The operational knowledge of skilled technicians gained from years of experience is invaluable for an enterprise. Possession of such knowledge will facilitate an enterprise sharing technician's know-how and training of new employees effectively. However, until now there is rare efficient quantitative method to obtain this kind of tacit knowledge. In this paper we propose a concept of engineering-oriented operational empirical knowledge (OEK) to describe this kind of knowledge and design a framework to acquire OEK from skilled technician's operations. The framework integrates motion analysis, motion elicitation, and intent analysis. The modular arrangement of predetermined time standards (MODAPTS) is used to divide the technician's operational process into basic motion elements; and the variable precision rough set (VPRS) algorithm is used to extract the technician's OEK content, which combined with the technician's intent elicited via interview; the completed OEK is obtained. At the end of our study, an engineering case is used to validate the feasibility of the proposed method, which shows that satisfactory results have been reached for the study.

\section{Introduction}

Years of experience in an operational work can bring a technician much know-how for accomplishing the tasks efficiently. The operational know-how of the skilled technician is of great importance for an enterprise, which often has new workers to train and need to update the workflow to increase productivity $[1,2]$. With the operational know-how transferred to novice, the novice can improve his operational skills and then increase manufacturing productivity and product quality. However, the operational know-how has not been sufficiently harnessed due to the lack of an efficient knowledge acquisition method [3-5]. Therefore, it is imperative to develop an efficient acquisition method for such knowledge acquisition.

Operational know-how is regarded as a kind of tacit knowledge, which is hard to formalize, communicate, and share with others [6]. The transformation from tacit knowledge to explicit knowledge is a challenging task. Moreover, many of tacit knowledge acquisition approaches are based on the literal documents, for the documents might be suitable for explanation of procedure. However, it is very rare to explain the secret of the technical operation, which the skilled technicians have. The acquisition of operational know-how is more difficult compared to the acquisition of tacit knowledge based on the literal documents.

In the existing literatures, apprenticeship and observation are the most useful methods to acquire operational know-how [3]. Apprenticeship is formal arrangements where specialized knowledge or skill from a domain expert is passed to a novice. The novice can acquire the same level of competence as the expert after extensively practicing the skill for a prescribed period of time [7]. Observation is also recognized as a valuable form of acquiring operational knowledge [8]. By observing the actions of an expert, the observer gains insights into expert's practices and builds his personal knowledge base. For example, in order to obtain an experienced baker's operating technique, the employees of Panasonic worked together with the skilled baker for a long time, observing and imitating the baker's motion. Eventually, the employees obtained the baker's operational knowhow. However, apprenticeship and observation belong to the 
qualitative methods. The disadvantages of these methods are obvious: they are inefficient, time-consuming, and not replicable, for different acquisition results will occur due to different cognition and learning modes of observers. Therefore, development of an efficient and quantitative method to elicit operational know-how becomes an emergent task in the field of knowledge management.

Aiming at the obstacles in the field of engineeringoriented operational know-how acquisition, we propose a concept of engineering-oriented operational empirical knowledge (OEK) to describe this kind of knowledge and then present an explicit definition and the representation of engineering-oriented OEK. A novel OEK acquisition (OEKA) framework is provided in our study, which can complete the transformation from technician's operational motion to the structured OEK. The OEKA integrates motion analysis, motion elicitation, and intent analysis. First, the worker's specific operation process is divided into basic motion elements by means of modular arrangement of predetermined time standards (MODAPTS), which is a system used to analyze the performance of manual work [9]. Second, we combine the motion elements with scenario factors to establish the scenario model, and then the algorithm of variable precision rough set (VPRS) is used to extract the critical motion of skilled technicians. Third, the skilled technician's intent behind the OEK content is elicited with the aid of interview. At last, the structured representation of OEK is constructed in the postprocessing.

Our contribution in this work is threefold:

(1) We define the concept of engineering-oriented OEK and provide the structured representation of OEK.

(2) We propose an integrated OEK acquisition framework integrating motion analysis, motion elicitation, and intent analysis, by which empirical knowledge in the engineer's operation can be obtained.

(3) We demonstrate that the extracted OEK can capture the technical know-how and can be used for novices' skill training.

The rest of the paper is organized as follows: we first review the related works in Section 2. In Section 3, we define the concept of OEK and then provide the representation model of OEK. In Section 4, we present the OEK acquisition method and its advantage. Section 5 describes the complete process of OEK acquisition. Section 6 provides a detailed case study. The comparison of our method with some related works is discussed in Section 7. Conclusions and future studies are outlined in the last section.

\section{Related Works}

2.1. Tacit Knowledge and Tacit Knowledge Acquisition. Knowledge tied to the senses, physical experiences, movement skills, intuition, unarticulated mental models, or implicit rules of thumb is tacit $[18,19]$. Tacit knowledge is rooted in the action, procedures, routines, ideals, commitment, and emotion, which makes it differ from the explicit knowledge that can be uttered and obtained as drawing and writing. Individuals with tacit knowledge are often unaware of what they possess and how valuable they are for others.

Tacit knowledge acquisition means eliciting tacit knowledge from various knowledge sources and representing it in the right form in order to share the knowledge among organizational members and reuse it $[20,21]$. Up till now, many tacit knowledge extraction methods have been developed and applied in the fields of software engineering, manufacturing engineering, and health care [22, 23]. Many of these methods are based on literal documents. And with the development of natural language processing machine learning, the tacit knowledge acquisition methods based on document are more and more efficient and valuable.

Although the methods based on literal document are suitable for acquiring the explanation of procedures, they are difficult to acquire the essence of the operational knowhow [24]. In addition, traditional knowledge extraction techniques work well in eliciting tacit knowledge in literal documents. They do not elicit the intent of the documents. However, intent is an indispensable part of tacit knowledge; the intent elicitation should be integrated in the tacit knowledge acquisition method [25].

2.2. Operational Know-How and Knowledge Acquisition. Operational know-how is regarded as a kind of tacit knowledge [5]. Such knowledge does not originate from the literature or documents identified as explicit knowledge but originates from the experience of an individual [26]. Consequently, it is difficult to be expressed and transferred $[27,28]$.

Many scholars have committed to obtaining the operational know-how and proposed many acquisition models or methods. In Table 1, the existing literatures about operational know-how acquisition are analyzed from the perspectives of method description, qualitative or quantitative, object of extraction, applied technology, application field, and drawback.

These methods are divided into three types: qualitative method, quantitative method, and mixed method.

Observation and apprenticeship are classic qualitative methods for extracting the operational know-how of skilled technicians [3]. Observation is recognized as a valuable form of learning according to the social cognitive theory [8]. The observer gains insights into the skilled technicians' practices and builds his personal knowledge base by observing the actions of the expert [29]. Apprenticeship is formal arrangements where specialized knowledge or skill from a domain expert is passed to a novice. The novice will acquire the same level of competence as the expert after extensively practicing the skill for a prescribed period of time [7]. These qualitative acquisition methods are successful at transferring the operational know-how from experts to novices and are the focus of earlier literatures. However, these methods have obvious drawbacks, such as the time-consuming and the inconsistent acquisition results for the different cognition and learning modes of learners.

The quantitative method of operational know-how acquisition is another type, in which the specific algorithm or tool is used to elicit the operational know-how. Nakagawa 
TABLE 1: Related literatures' analysis.

\begin{tabular}{|c|c|c|c|c|c|c|}
\hline Literature & $\begin{array}{c}\text { Method } \\
\text { description }\end{array}$ & $\begin{array}{l}\text { Qualitative or } \\
\text { quantitative }\end{array}$ & $\begin{array}{r}\text { Object of } \\
\text { extraction }\end{array}$ & $\begin{array}{c}\text { Applied } \\
\text { technology }\end{array}$ & Application field & Drawback \\
\hline $\begin{array}{l}\text { Bandura [8] and } \\
\text { Nonaka [10] }\end{array}$ & Observation & $\begin{array}{l}\text { Qualitative } \\
\text { method }\end{array}$ & $\begin{array}{c}\text { Actions of } \\
\text { domain expert }\end{array}$ & Observe & Manual work & $\begin{array}{l}\text { Inconsistent } \\
\text { acquisition } \\
\text { results }\end{array}$ \\
\hline Collis et al. $[7,11]$ & Apprenticeship & $\begin{array}{l}\text { Qualitative } \\
\text { method }\end{array}$ & $\begin{array}{c}\text { Specialized } \\
\text { knowledge or } \\
\text { skill of domain } \\
\text { expert }\end{array}$ & $\begin{array}{l}\text { Extensively } \\
\text { practicing the } \\
\text { skill }\end{array}$ & Not limited & $\begin{array}{c}\text { Time- } \\
\text { consuming and } \\
\text { inconsistent } \\
\text { acquisition } \\
\text { results } \\
\end{array}$ \\
\hline $\begin{array}{l}\text { Hashimoto et al. } \\
\text { [12] and Yoshida et } \\
\text { al. [5] }\end{array}$ & $\begin{array}{c}\text { Personal motion } \\
\text { sensing }\end{array}$ & Mixed method & $\begin{array}{c}\text { Skilled } \\
\text { operations of } \\
\text { skilled } \\
\text { technicians }\end{array}$ & $\begin{array}{l}\text { Field-oriented } \\
\text { interview, } \\
\text { human motion } \\
\text { capture, and } \\
\text { video analysis } \\
\end{array}$ & $\begin{array}{l}\text { Manufacturing } \\
\text { industry }\end{array}$ & $\begin{array}{l}\text { Depends on the } \\
\text { interview } \\
\text { seriously }\end{array}$ \\
\hline Watanuki [13] & $\begin{array}{c}\text { Method of skills } \\
\text { transfer }\end{array}$ & $\begin{array}{l}\text { Qualitative } \\
\text { method }\end{array}$ & $\begin{array}{c}\text { Casting } \\
\text { technologies } \\
\text { and skills of } \\
\text { skilled } \\
\text { technicians }\end{array}$ & $\begin{array}{l}\text { Sharing a "ba" } \\
\text { (place) using } \\
\text { multimedia } \\
\text { technology and } \\
\text { virtual reality } \\
\text { technology }\end{array}$ & $\begin{array}{c}\text { Manufacturing } \\
\text { skills training }\end{array}$ & $\begin{array}{c}\text { The } \\
\text { representation } \\
\text { of knowledge is } \\
\text { vague }\end{array}$ \\
\hline $\begin{array}{l}\text { Nakagawa et al. } \\
\text { [14] and Huang et } \\
\text { al. [15] }\end{array}$ & $\begin{array}{l}\text { Skill education } \\
\text { service }\end{array}$ & $\begin{array}{l}\text { Quantitative } \\
\text { method }\end{array}$ & $\begin{array}{l}\text { Extract skills } \\
\text { from nursing } \\
\text { motion (focused } \\
\text { on bed care } \\
\text { motion) }\end{array}$ & $\begin{array}{l}\text { Nursing skill is } \\
\text { extracted from } \\
\text { interview and } \\
\text { video analysis. } \\
\text { The extracted } \\
\text { skills are } \\
\text { assessed } \\
\text { quantitatively } \\
\end{array}$ & $\begin{array}{l}\text { Nurse and care } \\
\text { worker }\end{array}$ & $\begin{array}{l}\text { It is difficult to } \\
\text { formally } \\
\text { represent such } \\
\text { knowledge }\end{array}$ \\
\hline $\begin{array}{l}\text { Li et al. [16] and } \\
\text { Nguyen and Zhang } \\
{[2]}\end{array}$ & $\begin{array}{c}\text { Mobility and } \\
\text { physical activity } \\
\text { sensing }\end{array}$ & Mixed method & $\begin{array}{l}\text { Nurses, } \\
\text { firefighters, } \\
\text { waiters, } \\
\text { housekeepers, } \\
\text { or janitors }\end{array}$ & $\begin{array}{c}\text { Extract } \\
\text { high-level } \\
\text { knowledge rules } \\
\text { from low level } \\
\text { sensor signals } \\
\text { by means of an } \\
\text { unsupervised } \\
\text { approach } \\
\end{array}$ & Service & $\begin{array}{l}\text { Aiming at the } \\
\text { activities of } \\
\text { person; lacking } \\
\text { detailed motion }\end{array}$ \\
\hline $\begin{array}{l}\text { Mitsuhashi et al. } \\
\text { [17] }\end{array}$ & $\begin{array}{l}\text { Motion curved } \\
\text { surface analysis } \\
\text { and composite }\end{array}$ & $\begin{array}{l}\text { Quantitative } \\
\text { method }\end{array}$ & Skill succession & $\begin{array}{l}\text { Track the whole } \\
\text { joint motion by } \\
\text { means of } \\
\text { motion curved } \\
\text { surfaces and } \\
\text { confirm the } \\
\text { validity of skill } \\
\text { succession by } \\
\text { skeleton motion } \\
\text { movie and } \\
\text { curved surface }\end{array}$ & $\begin{array}{c}\text { Sports and } \\
\text { entertainment } \\
\text { motion }\end{array}$ & $\begin{array}{l}\text { Lacking } \\
\text { accurate } \\
\text { description of } \\
\text { the motion } \\
\text { sequences }\end{array}$ \\
\hline
\end{tabular}

et al. [14] extracted nursing skills by analyzing the body, foot, and muscle activities of experts and nonexperts and then developed a novel skill education service for nursing. Mitsuhashi et al. [17] tracked the whole joint motion by means of motion curved surfaces and confirmed the validity of skill succession by skeleton motion movie and curved surface. This method was used to elicit the know-how of sports and entertainment motion. The quantitative method can analyze the motion and behavior of person accurately.
However, the empirical knowledge representation in the method is somewhat weak.

The mixed method is an integrated framework including qualitative and quantitative methods. Yoshida et al. [5] presented a mixed method to extract the tacit knowledge consisting of the skilled operations of skilled technicians in the manufacturing industry. The method comprises three aspects: the field-oriented interview, the human motion capture, and the video analysis. However, this method depends 


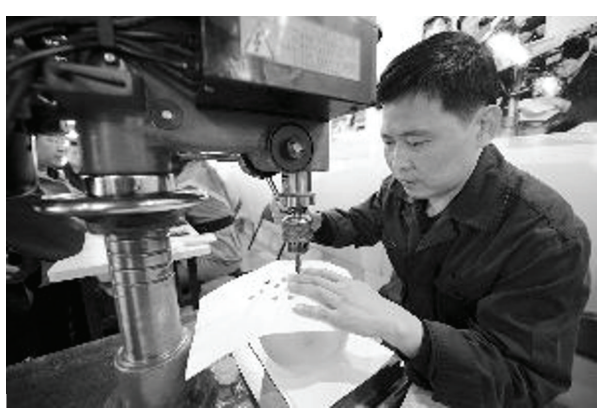

(a) Motion combanation

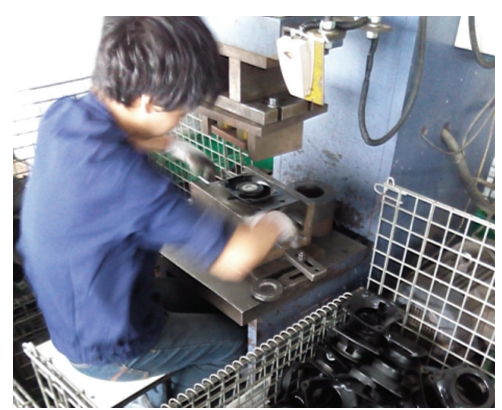

(b) Motion sequence

FIGURE 1: Examples of motion combanition and motion sequence.

on the interview seriously and may reduce the effectiveness of knowledge acquisition. Nguyen and Zhang [2] extracted high-level knowledge rules from low level sensor signals by means of an unsupervised approach in the service. Their study focused on the activities of person and lacked explicit motion description. The mixed method integrates the advantages of qualitative and quantitative methods and may be suitable to obtain the operational know-how of experts.

In summary, the explicit definition and representation of engineering-oriented operational knowledge are absent in the existing literatures. Such reasons have limited the development of standardized and effective knowledge acquisition approach. Our study aims to overcome these obstacles and present a novel and useful knowledge acquisition framework by means of mixed method. In the next section, the formal definition and representation of OEK are introduced.

\section{Definition and Representation of OEK}

3.1. Definition of OEK. In this paper, the engineeringoriented OEK is defined as follows.

Engineering-oriented OEK is the operational knowhow comprising the specific motion combination, motion sequence formed during the repeated practice, and thinking of an individual, which are of value for bettering the production process.

In the manufacturing process, some manual operations are very simple. For example, in the drilling operation, an operator only repeats the simple motion combination "pull and push," as shown in Figure 1(a). In other cases, the operation can be more complex. For example, in the molding operation shown in Figure 1(b), the operator uses his left hand to grasp a number of parts, placing them into a mold and rowing them against the mold, while his right hand operates the machine. When the molding is finished, the operator removes the completed artifact. Such a complex motion sequence includes a series of motion elements.

3.2. Interpretation of OEK Definition. Some key concepts in the definition of OEK are explained as follows.

(1) Operational Know-How Knowledge. Operational knowhow knowledge is the special knowledge that facilitates efficient engineering operations. This kind of knowledge does not originate from the literature or documents but originates from skilled technicians' experience [26].

(2) Motion Combination. Motion combination is the integration of a series of basic operational motion elements without pause. Motion combination is a basic form of the content of OEK. This kind of OEK comes from an individual's repeated practice and thinking, which are the most effective and convenient motions for the specific operation. For example, a proficient welding operator would use some particular motion combination in the welding process, which enables the operator to finish the production with high quality and quick speed.

The motion combination is formalized as follows:

$$
M_{c}=\left\{m_{1} m_{2} \cdots m_{n}\right\}
$$

where $M_{c}$ denotes the motion combination and $m_{n}$ is a basic motion element.

(3) Motion Sequence. A motion sequence is an ordered set of several motion combinations, where the pause between motion combinations is permitted. As another form of the content of OEK, the motion sequence represents a complete operation method, with which a skilled operator conducts the most effective operation process.

The motion sequence is formalized as follows:

$$
\begin{aligned}
M_{s}= & \left\{m_{a 1} m_{a 2} \cdots m_{a j}\right\} \vee\left\{m_{b 1} m_{b 2} \cdots m_{b k}\right\} \vee \cdots \\
& \vee\left\{m_{d 1} m_{d 2} \cdots m_{d l}\right\}
\end{aligned}
$$

where $M_{s}$ denotes the motion sequence, $m_{\text {in }}\{i=a, b, \ldots, d\}$ is a basic motion element, and $\left\{m_{a 1} m_{a 2} \cdots m_{a j}\right\}$ is a motion combination.

3.3. Representation of Engineering-Oriented OEK. Knowledge acquisition is closely related with knowledge representation, for the manipulation of acquired knowledge largely depends on how the knowledge is represented to reflect domain experts' mental model and problem solving process [30]. The main body of OEK representation is OEK content incorporating the motion combination and motion sequence 


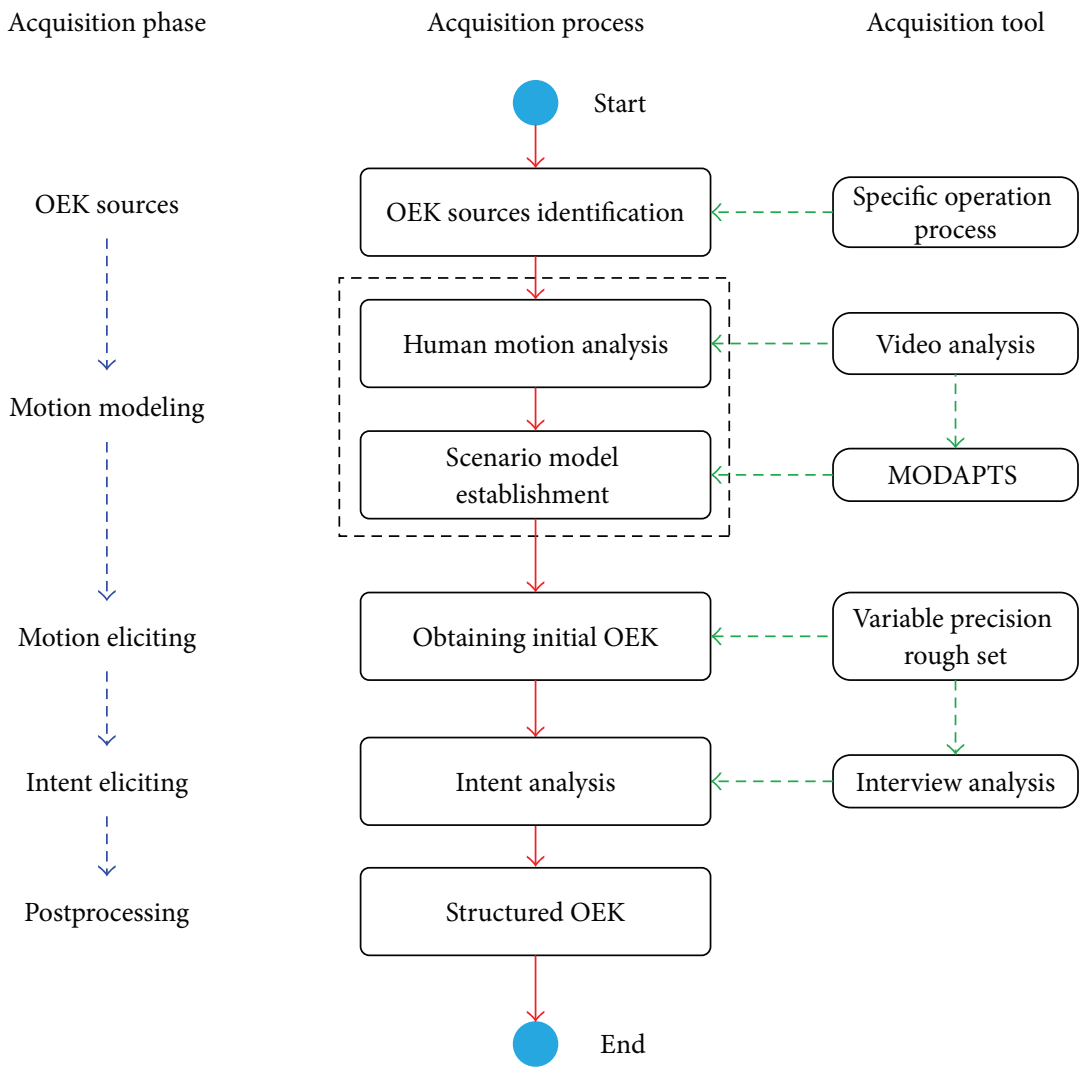

FIgURE 2: Multistage OEK acquisition framework.

obtained from a skilled technician. Besides this, since OEK is related with the specific operation process and problem scenarios, it is indispensable to integrate the scenarios into OEK representation [22, 23]. The operator's intent assumes a driving role of the motions, so it should be considered in the representation of OEK. Moreover, the OEK obtained from an individual experience is not always reliable, so a credibility score should be given to indicate the reliability of OEK. Taking the above factors into account, a complete OEK representation should include the problem scenarios, contributor's information, core content, intent, and creditability, as shown in the following formula:

$$
\begin{aligned}
& \langle\mathrm{OEK}\rangle=\langle\text { problem scenarios, contributor, content, } \\
& \text { intent, creditability }\rangle .
\end{aligned}
$$

\section{Methodology}

In many cases, skilled technicians are not aware of the OEK they possess and how this knowledge can be shared with others. In the problem solving, the technician's OEK is activated by the factors of problem context to realize the specific intent. In our study, not only the know-how in the motion but also the intent behind the specific motion will be elicited. The completed OEK is not obtained until the intents matching the specific actions are elicited, because it is difficult to finish the OEK elicitation process from the operator's practical action to the structured OEK in only one step; we present a novel multistage OEK acquisition framework as shown in Figure 2. The framework consists of five phases, which are (1) the stage of OEK sources identification, (2) the stage of motion analysis, (3) the stage of motion eliciting, (4) the stage of intent analysis, and (5) the stage of postprocessing.

The following knowledge acquisition techniques are used in the framework: human motion analysis, motion elicitation, and standard interview. In the previous literatures, each of them has been used separately. However, we construct a new data fusion analysis method which combines the three effectively and overcomes each one's weak point.

In the stage of OEK sources identification, the right manufacturing process or engineering problem is selected as the source of OEK acquisition.

In the stage of motion analysis, an engineer's operation in specific manufacturing process is captured and modeled. At first, an engineering problem in the manufacturing process is selected as the OEK source. And then the operator's operation is recorded by the video. At last, the motion and scenario model are established by means of MODAPTS [31].

The target of the stage of motion elicitation is to obtain the OEK content. The variable precision rough set algorithm is used to elicit such knowledge. 
In the stage of intent analysis, we use the standard interview to recognize the real intention of an expert's motion. The expert's motion matching the right intent will be retained.

In the postprocessing stage, the problem scenarios, contributor's information, and knowledge credibility are integrated with the OEK content. The structured OEK is acquired and the OEK acquisition process is finished.

\section{Description of OEK Acquisition Method}

5.1. Identification of OEK Sources. The aim of eliciting the OEK is to extract and store the skilled operator's knowhow and train the novice operators. The complex operational process difficult to grasp becomes the appropriate candidate of OEK sources. The skilled technicians' OEK in such process will be used to support novice training. Moreover, the workflows needing to be optimized to increase the productivity are also the potential candidate of OEK sources. The experts' OEK will be valuable to aid workflows redesign and optimization.

5.2. Motion Analysis and Modeling. The aim of this phase is to decompose the operation of operators into basic motion elements and then establish the motion and scenario model.

The operation is difficult to be accurately represented and quantitatively analyzed by direct observation [24]. The specific motion analysis tool is exploited to describe and explain the worker's operation [9]. The result of motion analysis can be used as the foundation of motion elicitation in the next stage.

There are four steps in this phase.

(1) Motion Capture. The motion capture is based on the stereo vision technique. It is introduced not only to acquire the time series posture data of engineers operating some equipment or machines but also to obtain the time varying relative position between the hand position and operated equipment [5].

In this step, firstly, stereo vision cameras are set on the appropriate place and calibrated. Secondly, the motions capture software on the PC to record the real operation of the operator. At the same time, shooting the operator by the video camera is started. It is important for video camera to take a position that does not disturb motion capture and cover the whole body of the expert engineer.

(2) Motion Modeling. In this step, the human motion is broken down into the basic motion elements based on the video shooting the operators. In production management field, the methods of motion analysis include therbligs, MTM (methods-time measurement) [32], MOST (Maynard operation sequence technique) [33], and MODAPTS (The modular arrangement of predetermined time standards).

The MODAPTS is a method used to analyze the performance of manual work [31]. In this paper, the MODAPTS is selected to conduct the motion analysis and modeling due to its advantages over others. First, MODAPTS, named "the language of work," is a useful tool of motion analysis, which can analyze the body motions required in a work task and the time those motions require in standard motion representation [34]. Second, MODAPTS is a simple, logic, effective, and low-cost motion analysis method and can be used with the aid of a desktop computer [35].

The MODAPTS has two distinguishing features: element motion classes, expressed in alphabetic form, and time values, expressed in units called "MODS." The movement class denoted as "Move" $(\mathrm{M})$ comprises motions done by body parts such as the finger, hand, or arm. The "terminal" class, comprising motions done at the end of an activity, consists of two kinds of activities: Get $(G)$ and Put $(P)$. The "auxiliary" class comprises motions that are not performed using body parts, such as walking, sitting, standing, deciding, and inspecting [36].

(3) Scenarios Modeling. In the field of knowledge management and knowledge engineering, constructing scenario models will facilitate knowledge acquisition and reuse [37]. Scenarios model can be used to describe the complexity of engineering problem solving process and is important foundation of knowledge sharing and reuse [38]. Scenarios usually have characteristic elements such as the presupposed setting, agents or actors, goals or objectives, and sequences of actions and events [39].

In our study, the scenarios model integrates processing goals and their subgoals and agents and their actions. In the scenarios model, agents and their actions representing the operators' motion combination or motion sequence are the essential elements and are used for eliciting skillful operators' OEK content during the next stage. The goal and subgoals in the model describe the different goals' level of process.

In the manufacturing process, the factors of "manmachine interaction" (scenario factors for short) should be considered in the scenarios model. The man-machine interaction explains the relationship between operator and machine. The special way of interaction between operators and machines or tools may be the potential technical knowhow of skillful operators, for example, the choice of tools and the preparation before processing. These factors do not belong to the motion combination or motion sequence and then are thorny to be described in the motion model. These factors are added to the scenario model.

The integrated scenarios model is shown in Figure 3. The model includes goals and subgoals of operation, agents and their actions, and story line. Specifically, the goal and subgoals describe the detailed production process. The agents and their actions represent the technician's operation, which can be used to elicit technician's OEK. The story line presents the sequence of operation; such information is often provided in the operation instructions.

(4) Establishing the Integrated Model. The information of product quality is added to the scenario model in this step. And then the integrated model is established as shown in Figure 4.

Up till now, the completed information including operational process and result is established in the integrated model, which can be used as the fundament of motion elicitation in the next stage. 


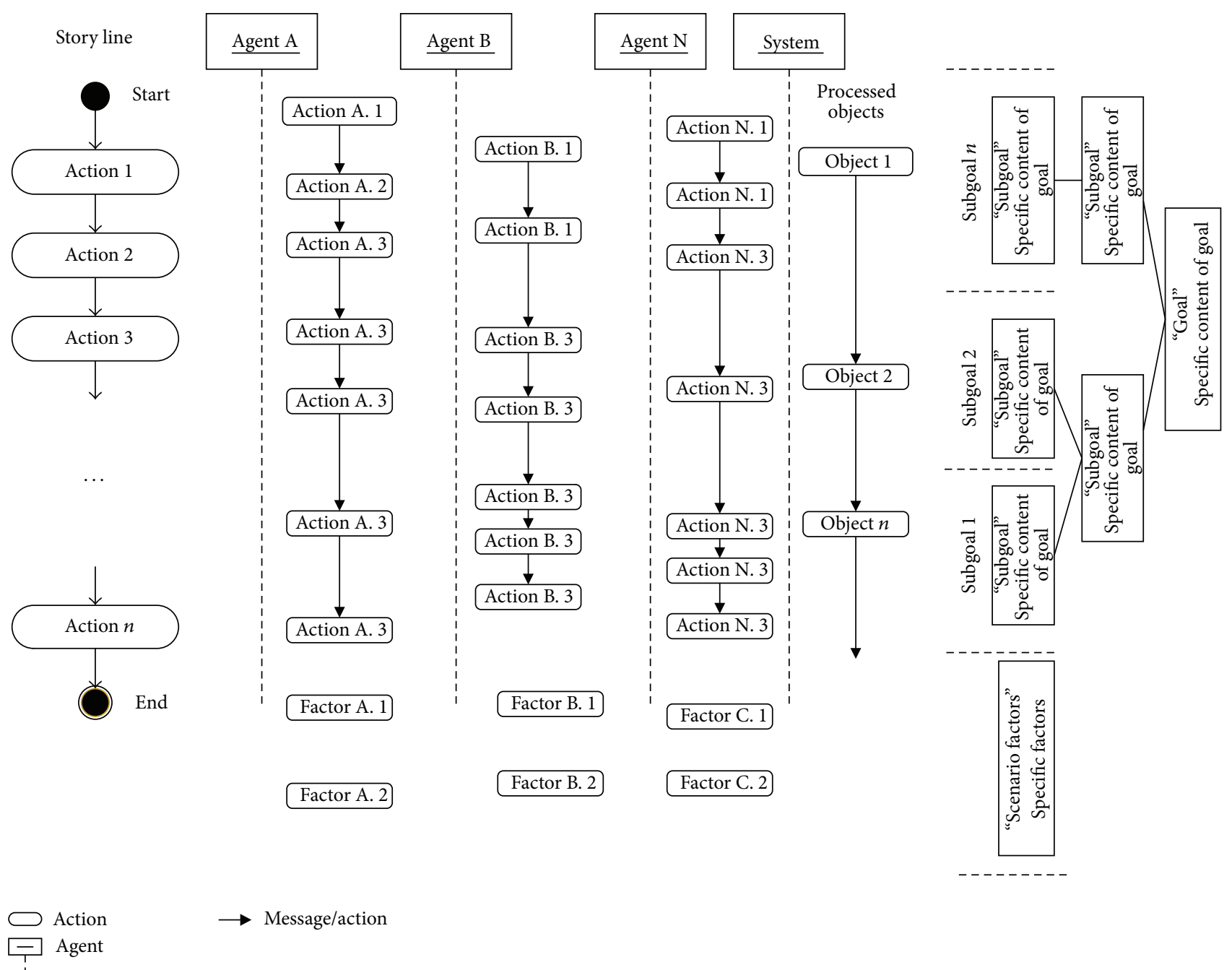

FIgURE 3: The scenario model.

5.3. Motion Elicitation. Motion elicitation is the critical stage in the OEK acquisition framework. The aim of motion elicitation is to obtain the expert's critical motion and scenario factors affecting product quality. The variable precision rough set (VPRS) is a useful tool of knowledge acquisition and is employed to elicit OEK in our study [40, 41].

As a mathematical methodology, rough sets provide a powerful tool for data analysis and knowledge discovery from imprecise and ambiguous data [42]. The rough sets theory has been successfully applied in diverse areas, such as knowledge acquisition, forecasting modeling, and data mining. The variable precision rough set (VPRS) model is an extension of the original rough set model. The main idea of VPRS is to allow objects to be classified with an error smaller than a certain predefined level [43].

5.3.1. Some Definitions Related to VPRS. VPRS operates on what may be described as a knowledge representation system or information system $[22,23,44]$. An information system is a 4-tuple $S=(U, A, V, f)$, where
$U$ is a nonempty finite set of objects;

$A$ is a nonempty finite set of attributes; we have $A=$ $C \cup D$ and $C \cap D=\varphi$, where $C$ is a nonempty set of condition attributes and $D$ is a nonempty set of decision attributes;

$V$ is the union of attribute domains; that is, $V=$ $\cup_{a \in A} V_{a}$, where $V_{a}$ is a finite attribute domain and the elements of $V_{a}$ are called value of attribute $a$;

$f: U \times A \rightarrow V$ is an information function which associates a unique value of each attribute with each object belonging to $U$; that is, $\forall a \in A$ and $\forall x \in U$; $f(x, a) \in V_{a}$.

Suppose that information system $S=(U, A, V, f)$, with each subset $Z \subseteq U$ and an equivalence relation $R$, referred to as an indiscernibility relation, corresponds to partitioning of $U$ into a collection of equivalence classes $U / R=$ $\left\{E_{1}, E_{2}, \ldots, E_{n}\right\}$, which represents that $U$ could be divided into $n$ subsets of $E_{1}, E_{2}, \ldots, E_{n}$ according to equivalence relation $R$. We will assume that all sets under consideration 


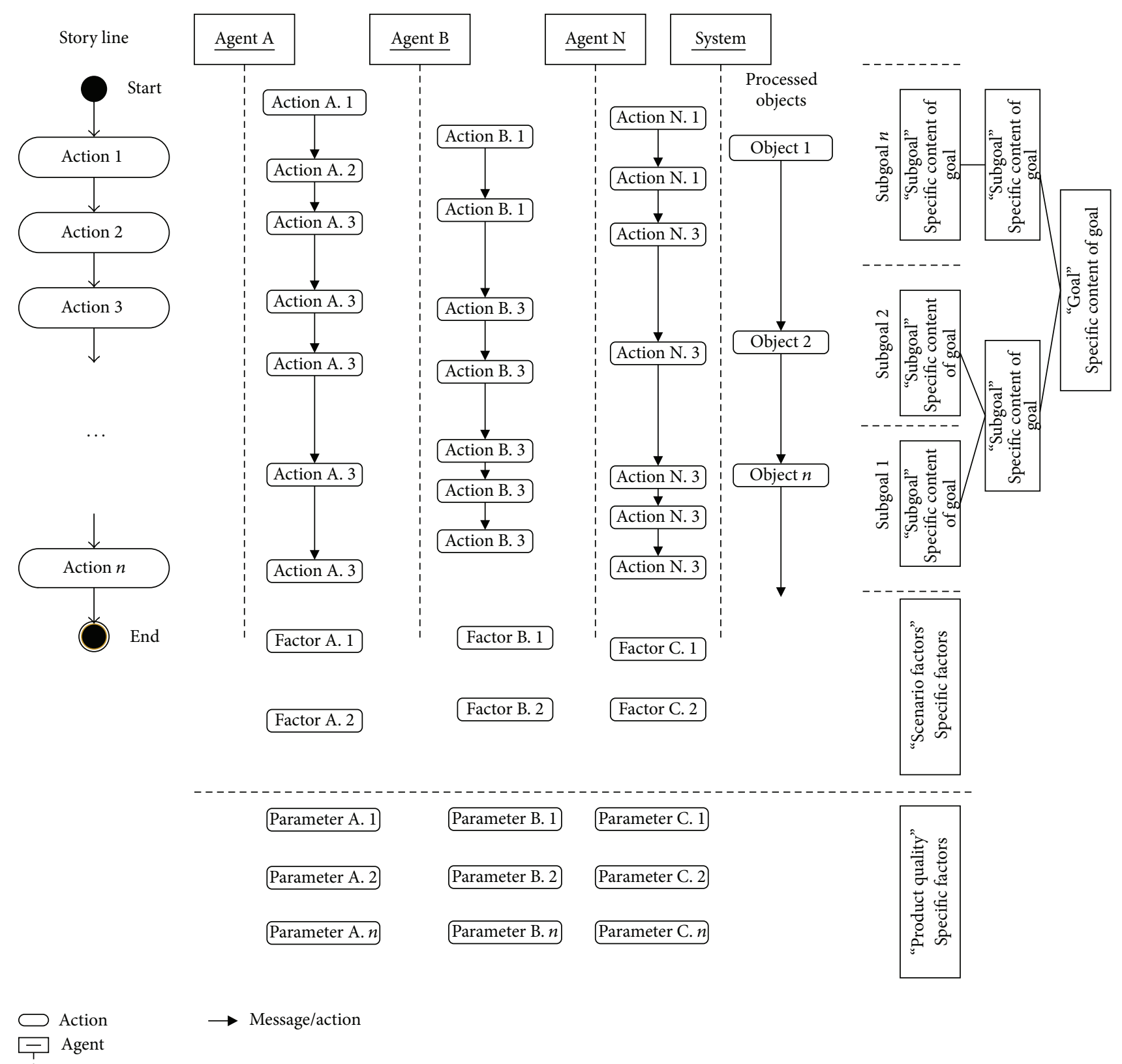

FIGURE 4: The integrated model.

are finite and nonempty. The variable precision rough sets approach to data analysis hinges on two basic concepts, namely, the $\beta$-lower and the $\beta$-upper approximations of a set. The $\beta$-lower and the $\beta$-upper approximations can also be presented in an equivalent form as shown below. The $\beta$-lower approximation of the set $Z \subseteq U$ and $P \subseteq C$ is

$$
\underline{C}_{\beta}(D)=\bigcup_{1-P_{r}\left(Z \mid x_{i}\right) \leq \beta}\left\{x_{i} \in E(P)\right\} .
$$
is

The $\beta$-upper approximation of the set $Z \subseteq U$ and $P \subseteq C$

$$
\bar{C}_{\beta}(D)=\bigcup_{1-P_{r}\left(Z \mid x_{i}\right)<1-\beta}\left\{x_{i} \in E(P)\right\},
$$

where $E(\cdot)$ denotes a set of equivalence classes (in the above definitions, they are condition classes based on a subset of attributes $P$ ). Consider

$$
\begin{gathered}
Z \subset E(D), \\
P_{r}\left(Z \mid x_{i}\right)=\frac{\operatorname{Card}\left(Z \cap x_{i}\right)}{\operatorname{Card}\left(X_{i}\right)} .
\end{gathered}
$$

Based on Ziarko [43], the measure of quality of classification for the VPRS model is defined as

$$
\gamma(P, D, \beta)=\frac{\operatorname{Card}\left(\bigcup_{1-P_{r}\left(Z \mid x_{i}\right)}\left\{x_{i} \in E(P)\right\}\right)}{\operatorname{Card}(U)},
$$


where $Z \subseteq E(D)$ and $P \subseteq C$, for a specified value of $\beta$. The value $\gamma(P, D, \beta)$ measures the proportion of objects in the universe $(U)$ for which a classification (based on decision attributes $D$ ) is possible at the specified value of $\beta$.

5.3.2. VPRS Knowledge Extraction Algorithm. The LEM2 algorithm proposed by Grzymala-Busse [45] is one of the most widely used algorithms and many real-world applications use this algorithm to extract knowledge. The VPRS knowledge extraction algorithm (VPRS-KE) originated from LEM2 and took the inclusion errors into account [46]. In our study, VPRS-KE is used to elicit the specific motion and scenario factors in the technician's operation.

In the VPRS-KE, a heuristic strategy is used in the algorithm to extract a minimum set of rules. All of positive regions and boundary region subsets can be denoted by $Y$, for each $K \in Y$ (the decision connecting with region $K$ is $D)$. Given that $C=c_{1} \wedge c_{2} \wedge \cdots \wedge c_{n}$ is the conjunction of $n$ elementary conditions, the objects in the decision table covered by $C$ can be expressed as follows:

$[C]$ is the cover of rule $C$ on the decision table.

$[C]_{K}^{+}=[C] \cap K$ is the positive cover of $C$ on $K$.

$[C]_{K}^{-}=[C] \cap(U-K)$ is the negative cover of $C$ on $K$.

A decision rule is denoted by $r$.

The procedure of the rule extraction is summarized in VPRS knowledge extraction algorithm as follows:

Input: $K$, each of the positive regions or the boundary regions subset in $Y:(K \in Y) ; \beta$ is the allowed inclusion error for VPRS.

Output: $R$, set of rules extracted from region $K$.

Step 1. $G \leftarrow K ; R \leftarrow \Phi$

Step 2. While $G \neq \Phi$

Step 3. Begin

Step 4. Initialize the condition set of a rule with an empty set: $C \leftarrow \Phi$

Step 5. Initialize $C_{\text {Current }}$ with all the attributes and their values in $G$ :

$$
C_{\text {Current }} \longleftarrow\{c:[c] \cap G \neq \Phi\}
$$

Step 6. Iteratively add conditions to $C$, until set $[C]$ is included in the set $K$ with an inclusion error threshold $\beta$ :

While $(C=\varphi)$ Or $(e([C], K) \leq \beta)$ do

Begin

Select $a \in C_{\text {Current }}$, such that $\operatorname{card}([a] \cap G)$ is maximum.

If ties occur, select $a$ with the smallest card([a]); and if further ties occur, select the first $a$ from the list;

Add condition $a$ to $C$

$$
C \longleftarrow C \cup\{a\}
$$

Eliminate the conditions which have been already used:

$$
C_{\text {Current }} \longleftarrow\{c:[c] \cap G \neq \Phi\}
$$

$$
\begin{aligned}
& \text { Update } C_{\text {Current }} \\
& \qquad C_{\text {Current }} \longleftarrow C_{\text {Current }}-C
\end{aligned}
$$

End

Step 7. Delete the redundant conditions:

For each $a \in C$ do

$$
\begin{gathered}
\text { If } e([C-\{a\}], K) \leq \beta \\
\text { Then } C \longleftarrow C-\{a\}
\end{gathered}
$$

End For

Step 8. Create a rule $r$ based on $C$, and put the rule into $R$ :

$$
R \longleftarrow R \cup\{r\}
$$

Step 9. Remove the objects covered by $R$ from $G$ :

$$
G \longleftarrow G-\bigcup_{r \in R}[r]
$$

Step 10. End

Step 11. Delete the redundant rules:

For each $r \in R$ do

$$
\text { If } K \subseteq \bigcup_{S \in(R-\{r\})}[s] \quad \text { Then } R \longleftarrow R-\{r\}
$$

End For

Step 12. Output $R$.

5.4. Intent Recognition. In cognitive psychology, intent refers to the thoughts one has before producing an action [47]. Intent recognition is to identify the goal of observed sequence of motions and is performed by the action agent. It is a process by which an agent can gain access to the goals of another and predict its future actions and trajectories. The understanding of human intent based on human motions remains a highly relevant and challenging research topic [48].

In our study, the standard interview is employed to detect the underlying intent behind human motion. First, the skilled operators are interviewed to evaluate OEK content and explain the specific intent behind the motion. Second, the advantage of skillful operations and the disadvantage of unskillful operations will be recognized.

5.5. Postprocessing. In the postprocessing, the related factors of problem scenarios, contributors, and creditability are integrated into the OEK content, and the completed representation of OEK is constructed. 
TABLE 2: MODAPTS representation of operator's welding process fragment.

\begin{tabular}{|c|c|c|c|c|}
\hline \multirow{2}{*}{ Number } & \multicolumn{2}{|c|}{ Motion of left hand } & \multicolumn{2}{|c|}{ Motion of right hand } \\
\hline & $\begin{array}{c}\text { Discomposed } \\
\text { motion description }\end{array}$ & $\begin{array}{l}\text { MOD } \\
\text { representation }\end{array}$ & $\begin{array}{c}\text { Discomposed } \\
\text { motion description }\end{array}$ & $\begin{array}{c}\text { MOD } \\
\text { representation }\end{array}$ \\
\hline 1 & Grasp the pipe & M4G1 & & \\
\hline 2 & $\begin{array}{l}\text { Insert the pipe and } \\
\text { adjust its position }\end{array}$ & M4P2 & $\begin{array}{l}\text { Prepare the } \\
\text { welding gun }\end{array}$ & M3P2 \\
\hline 3 & Hold the pipe & $\mathrm{H}$ & Start spot welding & $\mathrm{M} 1 \mathrm{P} 0 \times n$ \\
\hline
\end{tabular}

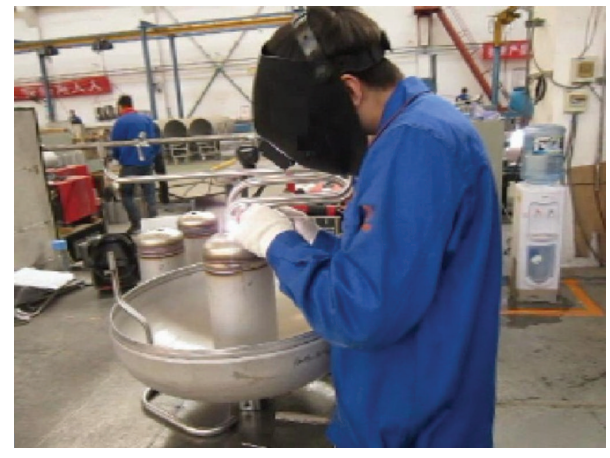

FIGURE 5: Shooting operator's operation.

\section{Case Study}

In our study, a manual welding case is used to illustrate the proposed OEK acquisition method. This case originates from a real manufacturing company's production improvement project.

6.1. Identification of OEK Sources. SQ company is a producer of new energy devices. The main product of company is LNG (Liquefied Natural Gas) cylinders. The welding process is widely used in the cylinders' manufacturing. The head of inner vessel, due to its complex internal structure, cannot be manufactured by autowelding. Therefore, manual welding is employed in this process (Figure 5).

In actual manufacturing, the varying quality of the product causes much trouble for the company. The major reason for the product's quality variation lies in the different welding methods used by the workers. Because the training of new employees needs a long time, there are always novices and skilled operators in the company. Although the company provides the standard operation instructions, the novices usually find it hard to grasp the technique know-how. For the skilled operators, they often feel difficult to tell what their empirical knowledge is and what kind of operation is the best in guiding the novice. Therefore, it is an urgent mission to analyze the operational know-how of the skilled operators and elicit their OEK.

\subsection{Motion Modeling}

Step 1 (motion capture). Fifteen skilled technicians and fifteen novices were invited to take part in the case. In this step, motion capture was carried out in the real operation situation as shown in Figure 5. It is important for the video camera to take a position that does not disturb the worker's operation. In our study, the wearable sensors were not chosen since they often burden the worker and affect human thinking [24].

Step 2 (motion analysis). The MODAPTS analysis can translate the welding operation into basic motion element class codes and time values through video examination [9]. For example, in an assembly process, the operator's motion sequence includes "move the left hand to the component," "get the component," "move the component," and "put the component," which are assigned the codes M3, G1, M3, and $\mathrm{P} 1$, respectively. Thus, the resulting motion MODAPTS is M3G1M3P1.

In our study, the welding process is represented alphanumerically as motion element sets by means of IEMS software. Figure 6 illustrates an example of a MODAPTS for sequential welding operations consisting of grasping, inserting, adjusting, and welding operations. Table 2 presents the MOD representation of a fragment of the operator's welding process.

Step 3 (establish scenario model). In this step, the result of MODAPTS analysis is used to construct the scenario model. The operator's motion combination and motion sequence are the main part of the scenario model. The goal and subgoals of the operator are the assistant part of scenario model.

The scenario factors are considered in the scenario model. In our case, the "man-machine interaction" includes two parts, the interaction between operator and objective and the interaction between operator and tools.

In the part of man-object interaction, the factors consist of the position of the welding arc starting point, the number of rotations welding a pipe, and the welding mode.

In the part of man-tool interaction, the factors include the welding gun posture in the difficult process part.

The scenario model is shown in Figure 7.

Step 4 (establish integrated model). The integrated model is established by attaching the quality information of each operation to the scenario model.

In the case, the quality of welding process is evaluated by the standard of the enterprise. The evaluation criteria include the weld surface color and the quality of the weld seam. The weld colors include white, yellow, and black. White is the best 


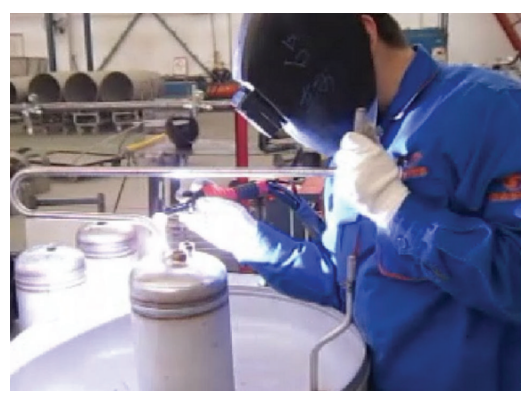

(a) A case of welding process
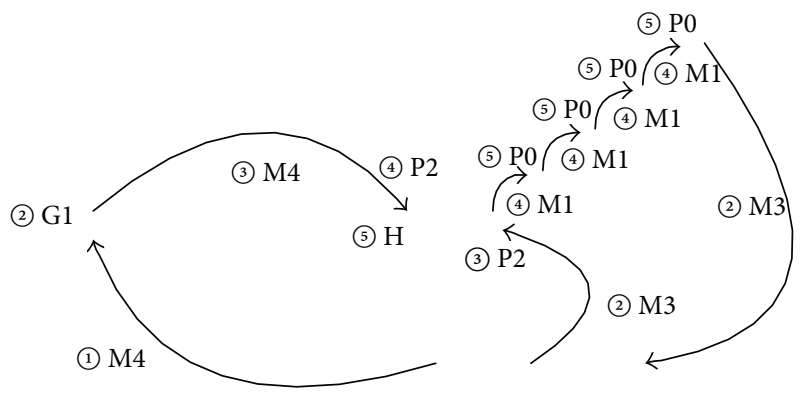

(b) A MODAPTS analysis of welding process

FIGURE 6: The MODAPTS analysis of a welding process fragment.

and black is the worst. In addition, the weld surface quality is divided into three classes: good, medium, and poor.

The integrated model is shown in Figure 8.

\subsection{OEK Content Eliciting}

(1) Preprocessing. The preprocessing is the fundamental of the eliciting algorithm in the next stage.

According to the characteristics of motion sequence and the requirement of VPRS algorithm, the following steps are conducted.

Step 1. Remove the meaningless motions, which have nothing to do with the operation, such as drinking and talking.

Step 2. Combine the repetitive motions, for example, combining $\mathrm{M} 2 \mathrm{P} 1 \mathrm{M} 2 \mathrm{P} 1 \mathrm{M} 2 \mathrm{P} 1$ into $\mathrm{M} 2 \mathrm{P} 1 \times 3$. If the repetitive time of motion combination is more than 3 , the time is represented as $n$.

The structured result of preprocessing is shown in Table 3. The explanation of header of Table 3 is as follows. ID indicates each operator. Steps indicate the subgoals in the operational process. Arcing point, the number of rotations, and mode choosing are scenario factors. Color and surface quality are the evaluation indices of welding process quality. In the content of table, null indicates no operation in the step.

(2) Eliciting Process. In this step, the initial OEK content is elicited by means of VPRS based on the result of preprocessing and is shown in Table 4.

6.4. Intent Analysis. In this case, the skilled technicians who participated in the experiment were interviewed to explain the specific intent behind the motion. The evaluation factors necessity and intent of the motions are listed in Table 5.

6.5. Postprocessing. In this stage, the related factors of problem scenarios, contributors, and creditability are integrated into the OEK content. A completed representation of the skilled operators' OEK is obtained from the above stages. The structured representation of OEK is shown in Table 6.

\section{Discussion}

7.1. Comparison of OEK between Skilled Technicians and Novices. The OEK obtained by our method is the specific know-how coming from the experience of skilled operator; such knowledge indicates the motional difference in the critical operational steps between skilled and unskilled operators. In this section, we will discuss such difference between skilled operators' OEK and novices' motions in order to evaluate the value of OEK.

We analyze the difference from two aspects of time consumption and energy consumption performance in the same operating procedures (Step 3, Step 5, and Step 6). We calculate the operators' time from the operation videos. In order to measure the energy consumption of different operators, CATIA, as leading human factors engineering software, is used as the platform to model the human motion and provide the energy consumption. Figure 9 is the CATIA simulation of different operators. The results are shown in Figure 10.

The difference of time consumption (15.6s and $10.5 \mathrm{~s}$ ) between skilled and unskilled operators is obvious according to Figure 10(a). However, to our surprise, the energy consumption of unskilled operators is more than three times that of skilled operators $(1190 \mathrm{cal}$ and $313 \mathrm{cal}$, see Figure 10(b)). The plausible explanation is the importance of OEK, which not only improves the product quality but also releases the operators' fatigue and then increases the operators' safety. Furthermore, the result of comparison demonstrates the accuracy of OEK acquired by our method.

7.2. Comparison of Our Method with Related Works. The evaluation framework of requirements of elicitation technique is used in our study. It is regarded as a reasonable and acceptable method to evaluate elicitation technique, although it is a qualitative evaluation method [49]. The evaluation framework includes four factors (elicitor, informant, problem domain, and elicitation process); and each factor includes some detailed attributes. In the evaluation framework, a compiled technique adequacy table is used to select the most adequate techniques for a specific application context [50]. In this section, the evaluation framework is revised and used to compare our study with related works in the context of 


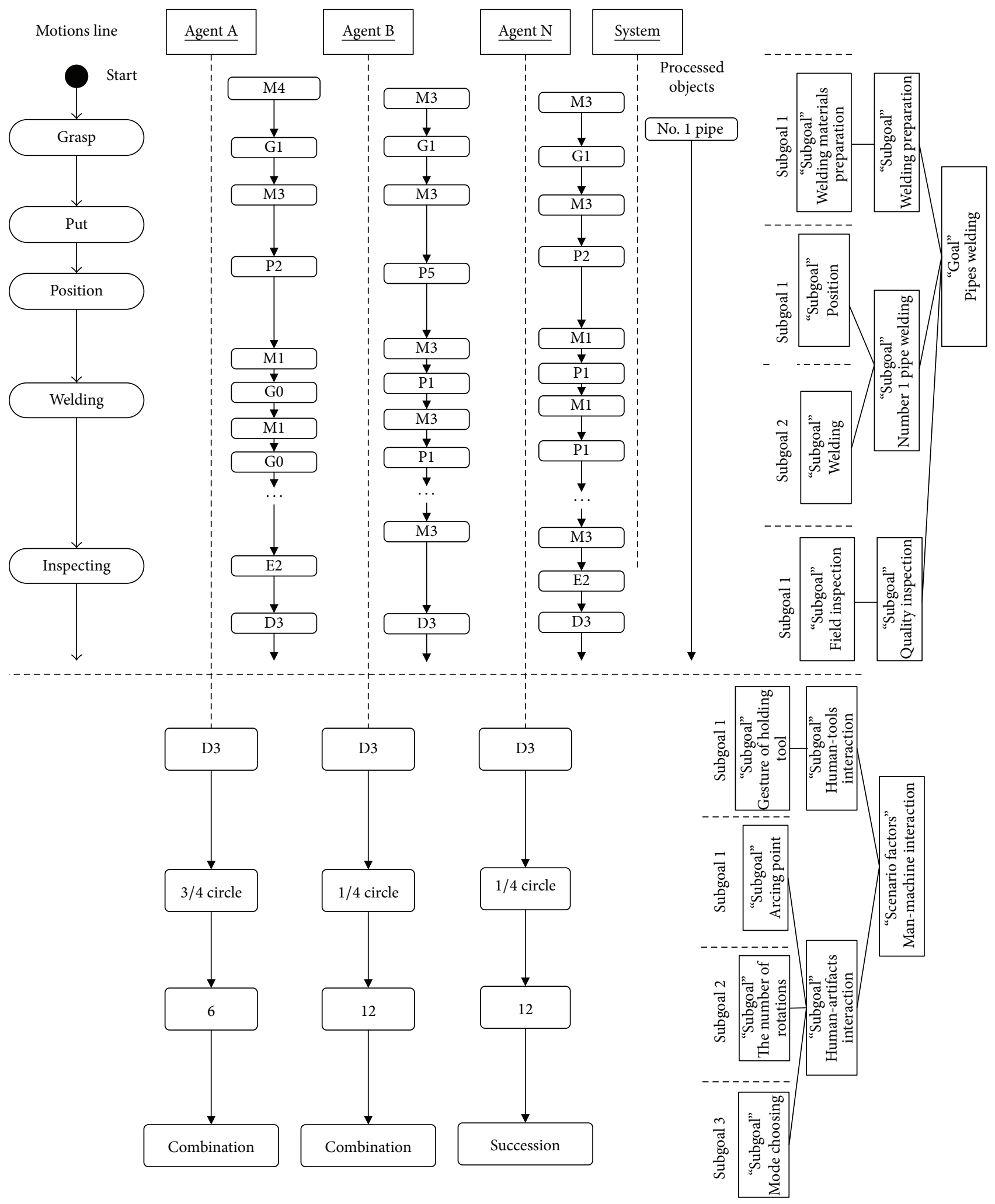

FIgURE 7: The scenario model of the case. 


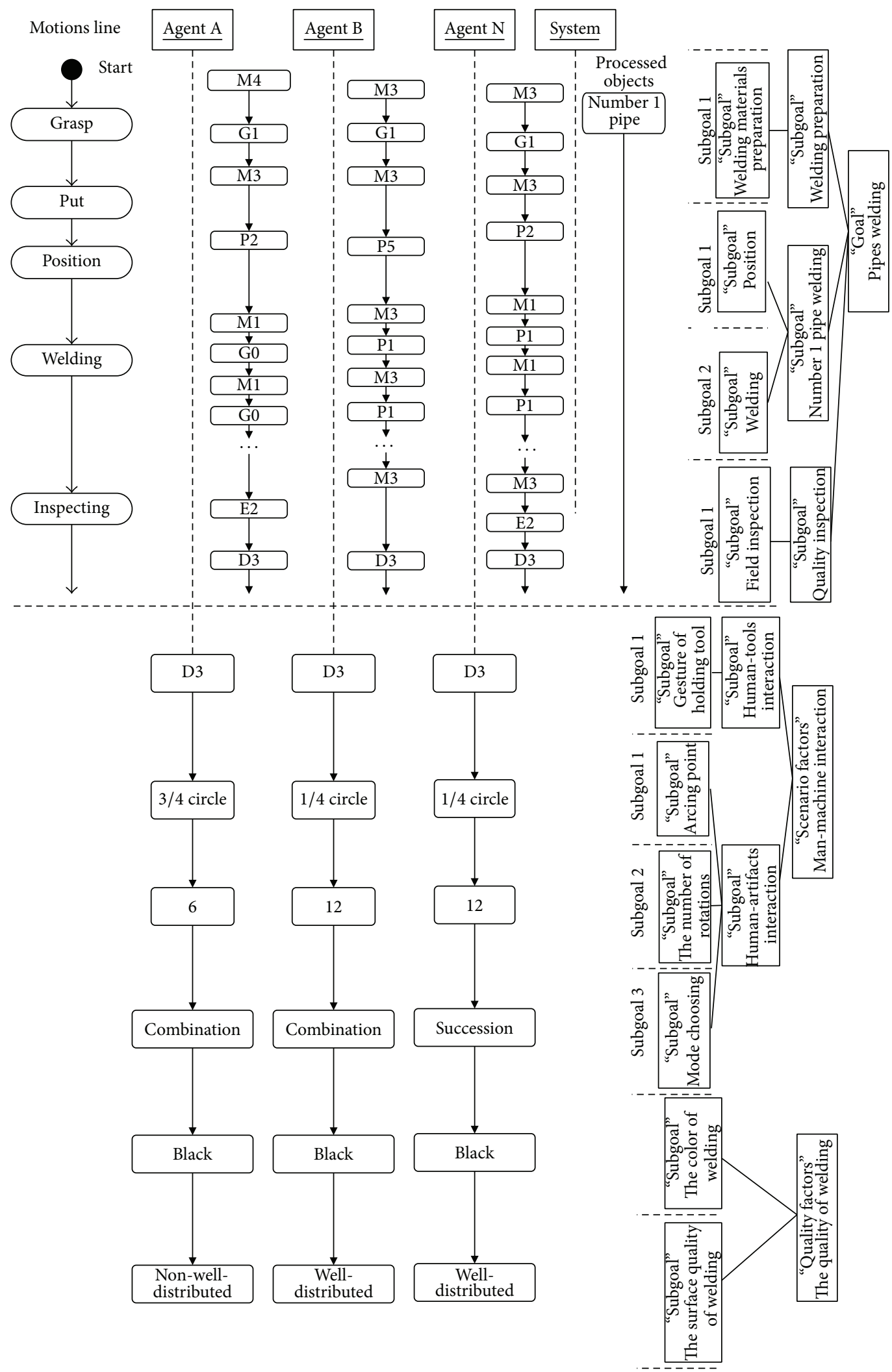

FIGURE 8: The integrated model of the case. 


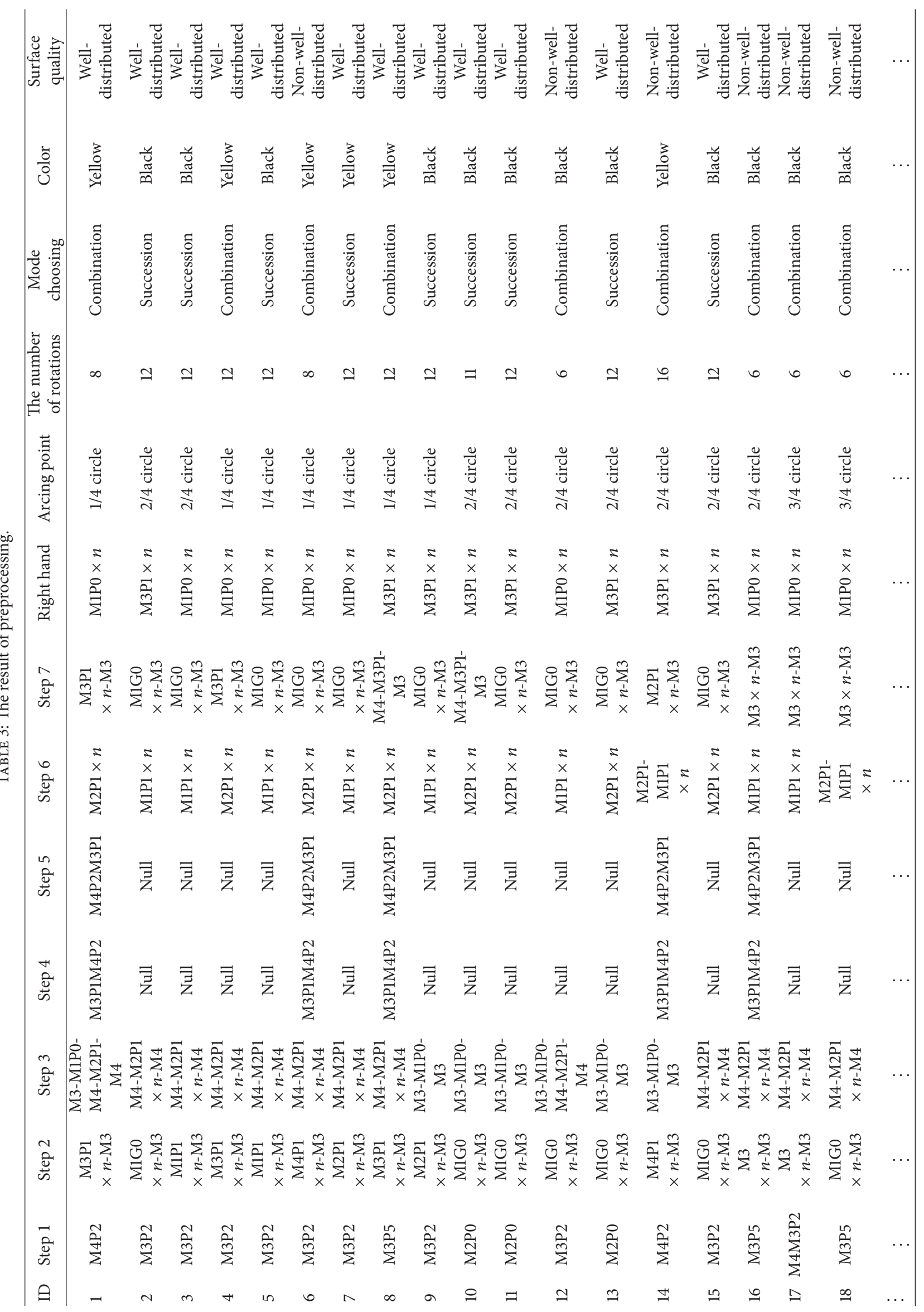




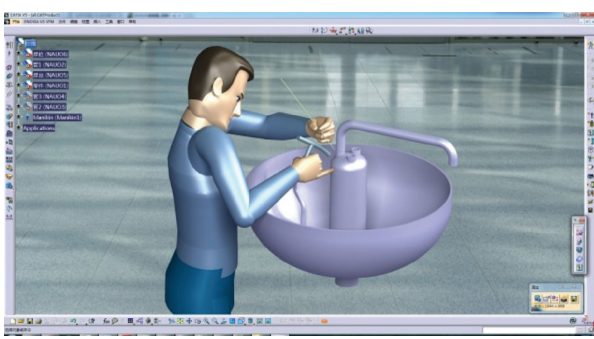

(a) Skilled technician's operation

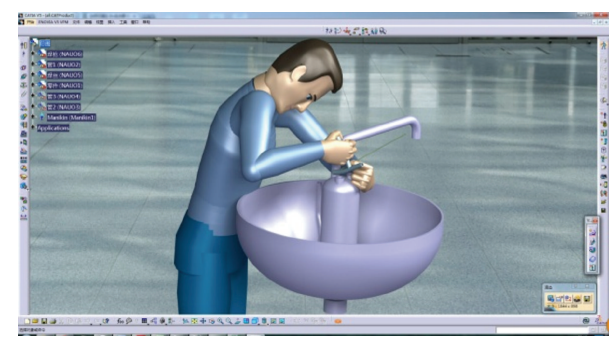

(b) Novice's operation

FIgURE 9: Operational simulation in CATIA.

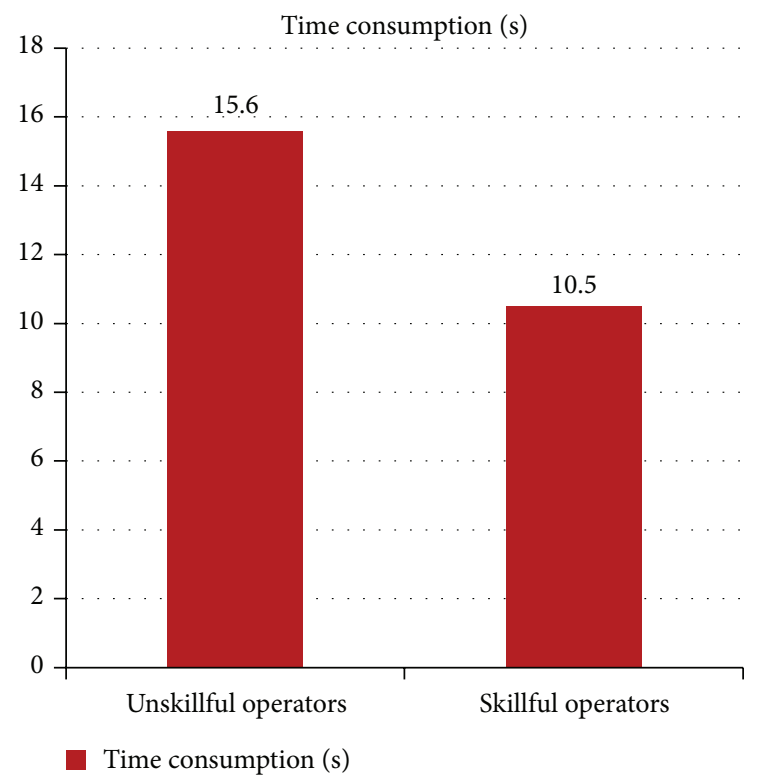

(a) Comparison of time consumption

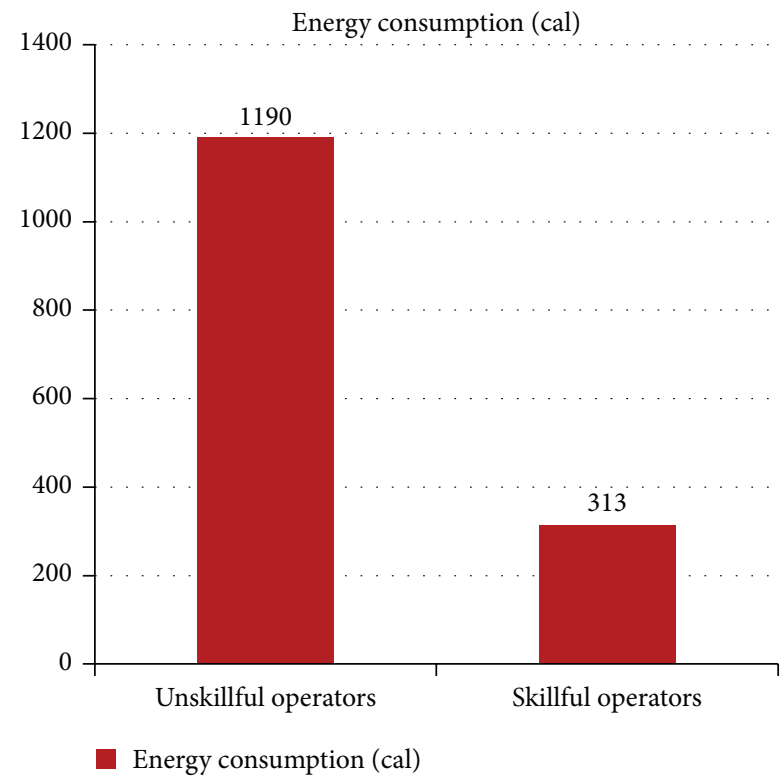

(b) Comparison of energy consumption

FIGURE 10: Comparison of time consumption and energy consumption.

engineering empirical knowledge elicitation. The advantage and adequacy level of our method under the context of engineering-oriented OEK acquisition are discussed as follows.

The classical qualitative methods (observation [8]), and apprenticeship [7]), a kind of quantitate method [14], and a kind of mixed method [5] are selected to be compared with our work. Three experts of knowledge management (two experts are university professors of China, and one expert is enterprise's senior manager) were invited to evaluate these methods using evaluation framework. The exhaustive description of factors is in the address http:// www.grise.upm.es/sites/extras/4/adequacy.pdf. The comparison among these methods is shown in Table 7.

In Table 7, the attribute values are expressed as the number of high, medium, and low values. Specifically, in the factors of elicitor and informant, the method with fewer requirements of person (elicitor and informant) is better. Accordingly, the number of low, medium, and high values is 2 , 1 , and 0 separately. However, in the factors of problem domain and elicitation process, the method with higher capabilities in the problem domain and elicitation process is better. Accordingly, the number of high, medium, and low values is 2,1 , and 0 separately, and the number of short, medium, and long values in the attribute of process time is 2,1 , and 0 . The evaluation result is shown in the column of total score. The performance of our method is best.

The performance of methods in each factor is explained in detail as follows. (1) The first factor is the factor of elicitor. This factor includes two attributes: requirement of elicitation techniques and domain knowledge. The medium elicitation techniques and domain knowledge are required in our method, for standard procedure is provided in our method. However, the superior elicitation techniques and domain knowledge are required in the qualitative methods and mixed methods, which rely on more interviews or observation, and thus require more elicitation techniques and domain knowledge. (2) The second factor is the factor of informant. Three attributes are in this factor: informant involvement in the elicitation process, personality of informant, and articulability of expert's empirical knowledge. In our method, the requirements of informant involvement 
TABLE 4: Initial OEK content.

\begin{tabular}{lccccccc}
\hline ID & \multicolumn{3}{c}{ Motion factors } & \multicolumn{2}{c}{ Scenario factors } & \multicolumn{2}{c}{ Product quality } \\
& Step 3 & Step 5 & Step 6 & Right hand & Arcing point & Color & Surface quality \\
\hline 001-LK & M3-M1P0-M4-M2P1-M4 & M4P2M3P1 & M2P1 $\times n$ & M1P0 $\times n$ & $1 / 4$ circle & Yellow & Well-distributed \\
002-YKB & M4-M2P1 $\times n$-M4 & M4P2M3P1 & M2P1 $\times n$ & M1P0 $\times n$ & $1 / 4$ circle & Yellow & Well-distributed \\
003-CSF & M4-M2P1 $\times n-M 4$ & M4P2M3P1 & M2P1 $\times n$ & M3P1 $\times n$ & $1 / 4$ circle & Yellow & Well-distributed \\
\hline
\end{tabular}

TABLE 5: Intent analysis of critical dimensions.

\begin{tabular}{lccccc}
\hline Factors & Support & Unrelated & Oppose & Conclusion & Effect description \\
\hline Step 3 & 10 & 0 & 0 & Useful & Obtain the high quality welds \\
Step 5 & 10 & 0 & 0 & Useful & Reduce the pause in the welding process \\
Step 6 & 10 & 0 & 0 & Useful & Easy to operate and control strength \\
Arcing point & 8 & 2 & 0 & Useful & Obtain the high quality welds \\
\hline
\end{tabular}

and communication with informant are less than those in other methods. In addition, articulability of expert's empirical knowledge in our method is medium. (3) The third factor is the factor of problem domain. Two attributes are in this factor: problem definedness and knowledge description. Our method has obvious advantage compared to other methods in this factor, because the explicit definition and representation of operational empirical knowledge of engineers are introduced in our study. These advantages can be recognized in the description of OEK acquisition method (Section 5) and case study (Section 6). (4) The fourth factor is the factor of elicitation process. Two attributes are in this factor: convenience of elicitation process and process time. Because of the explicit and standard knowledge acquisition process in our method, our method is more convenient than others in elicitation process. The preformation of our method in the process time is medium compared with other methods. In summary, our method has many advantages compared with other methods under the context of engineering operational knowledge elicitation.

\section{Conclusion}

8.1. Contribution. In our study, we propose a concept of engineering-oriented operational empirical knowledge (OEK) to describe the engineering-oriented operational know-how and provide the definition and representation of engineering-oriented OEK. A novel framework for acquiring engineering-oriented OEK from skilled technician's operations is presented, which can be used to elicit skilled technician's valuable, critical motion and intent. The framework constructs a completed knowledge acquisition process from skilled works' operation to the structured OEK.

The theoretical contribution of this study is multifold. First, few researches articulate the engineering-oriented OEK and present quantitative acquisition method for this kind of knowledge. Our study attempts to propose operational definition of engineering-oriented OEK and structured acquire framework, which is innovation in the field of knowledge acquisition. Our acquisition framework covers the completed tacit knowledge transfer process, while traditional methods tend to focus on the particular aspect or phase of tacit knowledge acquisition. Second, our research provides the fundamentals for tacit knowledge sharing and reusing.

The research findings provide some important application for practitioners. Our method uses "the language of work" to describe the technician's operational process and provide the exact representation of technician's operational know-how. In addition, the convenience and standardization of our method facilitate application in the manufacturing enterprise. The OEK obtained from skilled technician can be replicated and reused within novices.

8.2. Future Work and Limitation. There are some limitations in this study, which provide directions for future study.

One of the limitations is that knowledge engineers and technical professionals are involved in the knowledge acquisition process frequently. Too much human involvement may reduce the reliability of the acquisition method. However, some researchers argued that human involvement is indispensable for tacit knowledge acquisition. In the future, how to control the degree of human involvement in OEK acquisition will be studied. Moreover, the subtleties of human motion cannot be thoroughly described by the MODAPTS. In the future, how to identify the subtleties of human motion will be studied.

Though more and more manual operations have been replaced by robots, in some special manufacturing processes the manual working is still irreplaceable. Therefore, our study is valuable for manufacturing enterprises to acquire the critical operational know-how and improve the training of new employees.

\section{Competing Interests}

The authors declare that they have no competing interests.

\section{Acknowledgments}

The authors are most grateful to National Natural Science Foundation of China (nos. 70971085 and 71271133), the Research Fund for the Doctoral Program of Higher Education of China (no. 20100073110035), and Innovation Program 


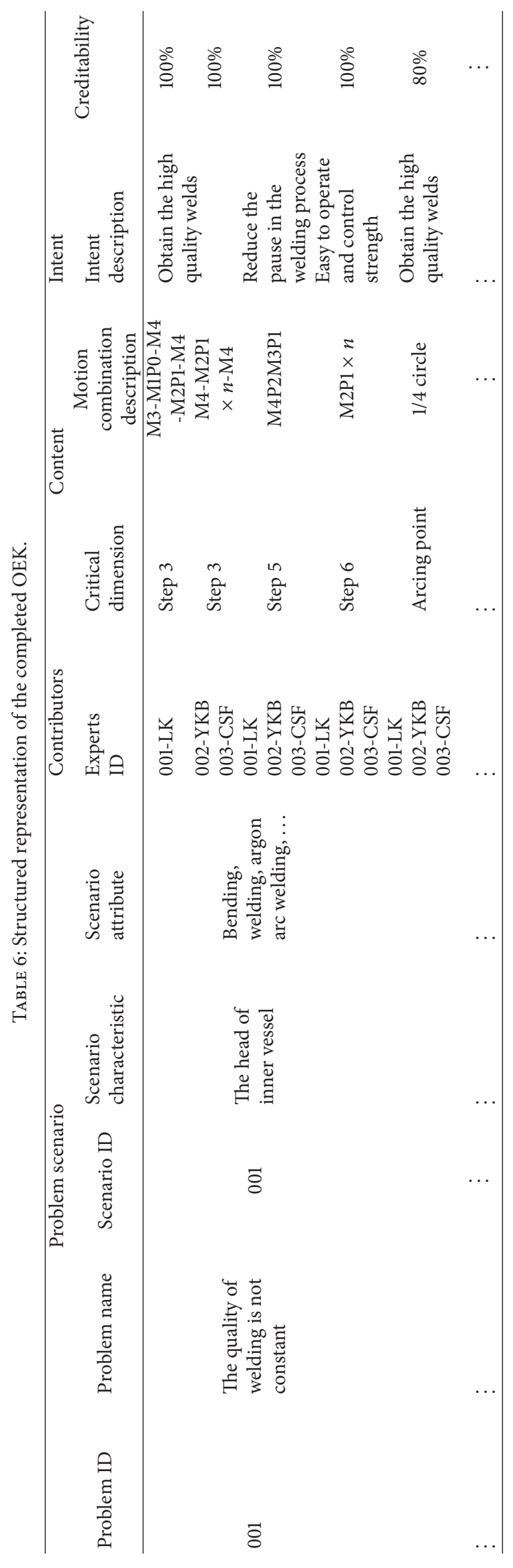


TABLE 7: The comparison between our study and other methods.

\begin{tabular}{|c|c|c|c|c|c|c|}
\hline \multicolumn{2}{|c|}{ Evaluation index } & \multirow[b]{2}{*}{ Values } & \multicolumn{4}{|c|}{ Methods } \\
\hline Factor & Attribute & & $\begin{array}{c}\text { Bandura [8] } \\
\text { and Collis } \\
\text { and Winnips } \\
{[7]}\end{array}$ & $\begin{array}{c}\text { Nakagawa et } \\
\text { al. [14] and } \\
\text { Huang et al. } \\
\text { [15] }\end{array}$ & $\begin{array}{c}\text { Yoshida et al. } \\
{[5]}\end{array}$ & This study \\
\hline \multirow[t]{2}{*}{ Elicitor } & $\begin{array}{c}\text { Requirements } \\
\text { of elicitation } \\
\text { techniques }\end{array}$ & Low/medium/high & M & M & $\mathrm{H}$ & M \\
\hline & $\begin{array}{l}\text { Knowledge of } \\
\text { (familiarity } \\
\text { with) domain }\end{array}$ & Low/medium/high & $\mathrm{H}$ & M & $\mathrm{H}$ & M \\
\hline \multirow{3}{*}{ Informant } & $\begin{array}{l}\text { Involvement } \\
\text { of informant }\end{array}$ & Low/medium/high & $\mathrm{H}$ & M & $\mathrm{H}$ & M \\
\hline & $\begin{array}{c}\text { Personality of } \\
\text { informant }\end{array}$ & Low/medium/high & $\mathrm{H}$ & M & M & $\mathrm{L}$ \\
\hline & $\begin{array}{l}\text { Articulability } \\
\text { of knowledge }\end{array}$ & Low/medium/high & $\mathrm{L}$ & M & M & M \\
\hline \multirow{2}{*}{$\begin{array}{l}\text { Problem } \\
\text { domain }\end{array}$} & $\begin{array}{l}\text { Knowledge } \\
\text { description }\end{array}$ & High/medium/low & $\mathrm{L}$ & M & $\mathrm{L}$ & $\mathrm{H}$ \\
\hline & $\begin{array}{c}\text { Problem } \\
\text { definedness }\end{array}$ & High/medium/low & $\mathrm{L}$ & $\mathrm{H}$ & M & $\mathrm{H}$ \\
\hline \multirow{2}{*}{$\begin{array}{l}\text { Elicitation } \\
\text { process }\end{array}$} & Convenience & High/medium/low & $\mathrm{L}$ & $\mathrm{M}$ & $\mathrm{M}$ & $\mathrm{H}$ \\
\hline & Process time & Short/medium/long & $\mathrm{L}$ & $\mathrm{L}$ & S & M \\
\hline \multicolumn{2}{|c|}{ Total score } & & 3 & 9 & 6 & 13 \\
\hline
\end{tabular}

of Shanghai Municipal Education Commission (13ZZ012) for financial supports that made this research possible.

\section{References}

[1] O. Brdiczka and V. Bellotti, "Identifying routine and telltale activity patterns in knowledge work," in Proceedings of the Fifth IEEE International Conference on Semantic Computing (ICSC '11), pp. 95-101, IEEE, Palo Alto, Calif, USA, September 2011.

[2] L. T. Nguyen and J. Zhang, "Unsupervised work knowledge mining through mobility and physical activity sensing," in Proceedings of the 6th International Conference on Mobile Computing, Applications and Services (MobiCASE '14), pp. 3039, IEEE, November 2014.

[3] A. Chennamaneni and J. T. C. Teng, "An integrated framework for effective tacit knowledge transfer," in Proceedings of the 17th Americas Conference on Information Systems 2011 (AMCIS '11), pp. 2471-2480, Detroit, Mich, USA, August 2011.

[4] A. Haug, "The illusion of tacit knowledge as the great problem in the development of product configurators," Artificial Intelligence for Engineering Design, Analysis and Manufacturing, vol. 26, no. 1, pp. 25-37, 2012.

[5] I. Yoshida, Y. Teramoto, H. Tabata, C. Han, and H. Hashimoto, "Tacit knowledge extraction of skillful operation from expert engineers," in Proceedings of the IEEE/ASME International Conference on Advanced Intelligent Mechatronics (AIM '11), pp. 554-559, IEEE, Budapest, Hungary, July 2011.

[6] R. K. Wagner and R. J. Sternberg, "Practical intelligence in real-world pursuits: the role of tacit knowledge," Journal of Personality and Social Psychology, vol. 49, no. 2, pp. 436-458, 1985.
[7] B. Collis and K. Winnips, "Two scenarios for productive learning environments in the workplace," British Journal of Educational Technology, vol. 33, no. 2, pp. 133-148, 2002.

[8] A. Bandura, Social Learning Theory, General Learning Press, New York, NY, USA, 1977.

[9] H. Cho, S. Lee, and J. Park, "Time estimation method for manual assembly using MODAPTS technique in the product design stage," International Journal of Production Research, vol. 52, no. 12, pp. 3595-3613, 2014.

[10] I. Nonaka, "A dynamic theory of organizational knowledge creation," Organization Science, vol. 5, no. 1, pp. 14-37, 1994.

[11] M. G. Sobol and D. Lei, "Environment, manufacturing technology, and embedded knowledge," International Journal of Human Factors in Manufacturing, vol. 4, no. 2, pp. 167-189, 1994.

[12] H. Hashimoto, I. Yoshida, Y. Teramoto, H. Tabata, and C. Han, "Extraction of tacit knowledge as expert engineer's skill based on mixed human sensing," in Proceedings of the 20th IEEE International Symposium on Robot and Human Interactive Communication (RO-MAN '11), pp. 413-418, IEEE, Atlanta, Ga, USA, August 2011.

[13] K. Watanuki, "A mixed reality-based emotional interactions and communications for manufacturing skills training," in Emotional Engineering, S. Fukuda, Ed., pp. 39-61, Springer, London, UK, 2011.

[14] J. Nakagawa, Q. An, Y. Ishikawa et al., "Extraction and evaluation of proficiency in bed care motion for education service of nursing skill," in Proceedings of the 2nd International Conference on Serviceology (ICServ '14), pp. 91-96, 2014.

[15] Z. Huang, A. Nagata, M. Kanai-Pak et al., "Automatic evaluation of trainee nurses' patient transfer skills using multiple kinect sensors," IEICE Transactions on Information and Systems, vol. 97, no. 1, pp. 107-118, 2014. 
[16] N. Li, A. J. Schreiber, W. Cohen, and K. Koedinger, "Efficient complex skill acquisition through representation learning," Advances in Cognitive Systems, no. 2, pp. 149-166, 2012.

[17] K. Mitsuhashi, H. Hashimoto, and Y. Ohyama, "Motion curved surface analysis and composite for skill succession using RGBD camera," in Proceedings of the 12th International Conference on Informatics in Control, Automation and Robotics (ICINCO '15), pp. 406-413, Alsace, France, July 2015.

[18] I. Nonaka and H. Takeuchi, The Knowledge-Creating Company: How Japanese Companies Create the Dynamics of Innovation, Oxford University Press, New York, NY, USA, 1995.

[19] I. Nonaka and R. Toyama, "The knowledge-creating theory revisited: knowledge creation as a synthesizing process," Knowledge Management Research \& Practice, vol. 1, no. 1, pp. 2-10, 2003.

[20] K. M. Ford, J. M. Bradshaw, J. R. Adams-Webber, and N. M. Agnew, "Knowledge acquisition as a constructive modeling activity," International Journal of Intelligent Systems, vol. 8, no. 1, pp. 9-32, 1993.

[21] W. Y. Zhang, S. B. Tor, and G. A. Britton, "A two-level modelling approach to acquire functional design knowledge in mechanical engineering systems," International Journal of Advanced Manufacturing Technology, vol. 19, no. 6, pp. 454-460, 2002.

[22] J. N. Liu, Y. Hu, and Y. He, "A set covering based approach to find the reduct of variable precision rough set," Information Sciences. An International Journal, vol. 275, pp. 83-100, 2014.

[23] L. Liu, Z. Jiang, and B. Song, "A novel two-stage method for acquiring engineering-oriented empirical tacit knowledge," International Journal of Production Research, vol. 52, no. 20, pp. 5997-6018, 2014.

[24] L. T. Nguyen and J. Zhang, "Unsupervised work knowledge mining through mobility and physical activity sensing," in Proceedings of the 6th International Conference on Mobile Computing, Applications and Services (MobiCASE '14), pp. 3039, IEEE, Austin, Tex, USA, November 2014.

[25] J. Liu and X. Hu, "A reuse oriented representation model for capturing and formalizing the evolving design rationale," Artificial Intelligence for Engineering Design, Analysis and Manufacturing, vol. 27, no. 4, pp. 401-413, 2013.

[26] S. Popadiuk and C. W. Choo, "Innovation and knowledge creation: how are these concepts related?" International Journal of Information Management, vol. 26, no. 4, pp. 302-312, 2006.

[27] S. S. R. Abidi, Y.-N. Cheah, and J. Curran, "A knowledge creation info-structure to acquire and crystallize the tacit knowledge of health-care experts," IEEE Transactions on Information Technology in Biomedicine, vol. 9, no. 2, pp. 193-204, 2005.

[28] L. Zhongtu, W. Qifu, and C. Liping, "A knowledge-based approach for the task implementation in mechanical product design," The International Journal of Advanced Manufacturing Technology, vol. 29, no. 9, pp. 837-845, 2006.

[29] D. Leonard-Barton, "Core capabilities and core rigidities: a paradox in managing new product development," Strategic Management Journal, vol. 13, no. S1, pp. 111-125, 1992.

[30] A. Abecker, S. Aitken, F. Schmalhofer, and B. Tschaitschian, "KARATEKIT: tools for the knowledge-creating company," in Proceedings of the Knowledge Acquisition for Knowledge-Based Systems Workshop (KAW '98), pp. 18-23, Banff, Canada, April 1998.

[31] G. C. Heyde, Modapts Plus, Heyde Dynamics, Sydney, Australia, 1983.
[32] D. W. Karger and F. H. Bayha, Engineered Work Measurement, The Industrial Press, New York, NY, USA, 1966.

[33] K. B. Zandin, MOST Work Measurement Systems, CRC Press, Boca Raton, Fla, USA, 2002.

[34] A. Freivalds and J. Goldberg, "Specification of base for variable relaxation allowance," Journal of Methods Time Measurement, no. 14, pp. 2-29, 1988.

[35] H. Cho and J. Park, "Motion-based method for estimating time required to attach self-adhesive insulators," CAD Computer Aided Design, vol. 56, pp. 68-87, 2014.

[36] B. W. Niebel, Motion and Time Study, Irwin, Homewood, Ill, USA, 8th edition, 1988.

[37] L. de Brabandere and A. Iny, "Scenarios and creativity: thinking in new boxes," Technological Forecasting and Social Change, vol. 77, no. 9, pp. 1506-1512, 2010.

[38] P. Brézillon, "Context in problem solving: a survey," Knowledge Engineering Review, vol. 14, no. 1, pp. 47-80, 1999.

[39] C. Y. Potts, "Using schematic scenarios to understand user needs," in Proceedings of the ACM 1st Conference on Designing Interactive Systems: Processes, Practices, Methods, \& Techniques (DIS '95), pp. 247-256, 1995.

[40] Y. Leung, W.-Z. Wu, and W.-X. Zhang, "Knowledge acquisition in incomplete information systems: a rough set approach," European Journal of Operational Research, vol. 168, no. 1, pp. 164-180, 2005.

[41] J.-S. Mi, W.-Z. Wu, and W.-X. Zhang, "Approaches to knowledge reduction based on variable precision rough set model," Information Sciences, vol. 159, no. 3-4, pp. 255-272, 2004.

[42] Z. Pawlak, "Rough sets," International Journal of Computer \& Information Sciences, vol. 11, no. 5, pp. 341-356, 1982.

[43] W. Ziarko, "Variable precision rough set model," Journal of Computer and System Sciences, vol. 46, no. 1, pp. 39-59, 1993.

[44] C.-T. Su and J.-H. Hsu, "Precision parameter in the variable precision rough sets model: an application," Omega, vol. 34, no. 2, pp. 149-157, 2006.

[45] J. W. Grzymala-Busse, "LERS—a system for learning from examples based on rough sets," in Intelligent Decision Support: Handbook of Applications and Advances of the Rough Sets Theory, R. Slowinski, Ed., pp. 3-18, Kluwer Academic, Dordrecht, The Netherlands, 1992.

[46] X. Pan, S. Zhang, H. Zhang, X. Na, and X. Li, "A variable precision rough set approach to the remote sensing land use/cover classification," Computers \& Geosciences, vol. 36, no. 12, pp. 1466-1473, 2010.

[47] D. M. Wegner, "Précis of the illusion of conscious will," Behavioral and Brain Sciences, vol. 27, no. 5, pp. 649-659, 2004.

[48] H. S. Kim, J. M. Kim, and W. G. Lee, "IE behavior intent: a study on ICT ethics of college students in korea," Asia-Pacific Education Researcher, vol. 23, no. 2, pp. 237-247, 2014.

[49] Y. Rana and A. Tamara, "An enhanced requirements elicitation framework based on business process models," Scientific Research and Essays, vol. 10, no. 7, pp. 279-286, 2015.

[50] D. Carrizo, O. Dieste, and N. Juristo, "Systematizing requirements elicitation technique selection," Information and Software Technology, vol. 56, no. 6, pp. 644-669, 2014. 


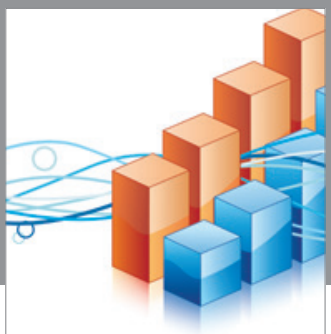

Advances in

Operations Research

vatem alat4

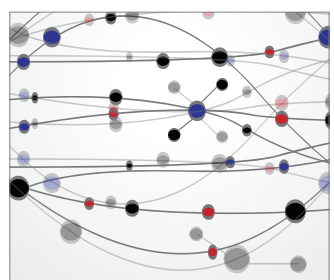

\section{The Scientific} World Journal
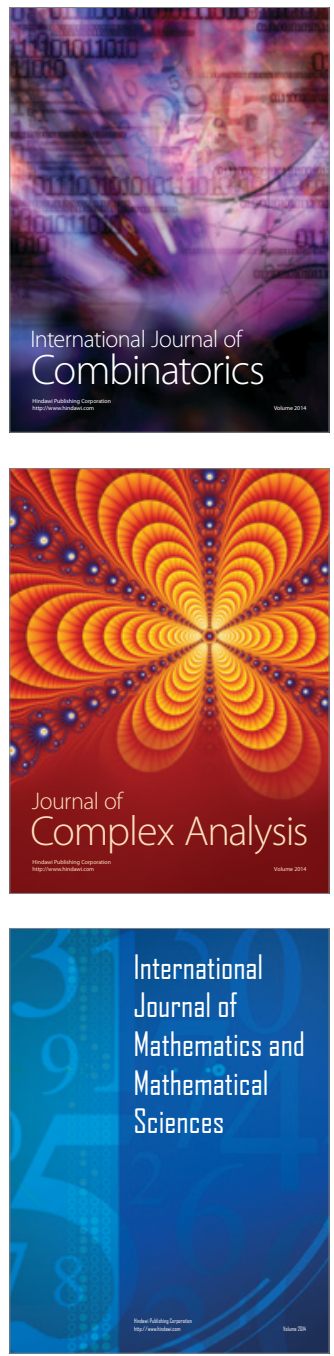
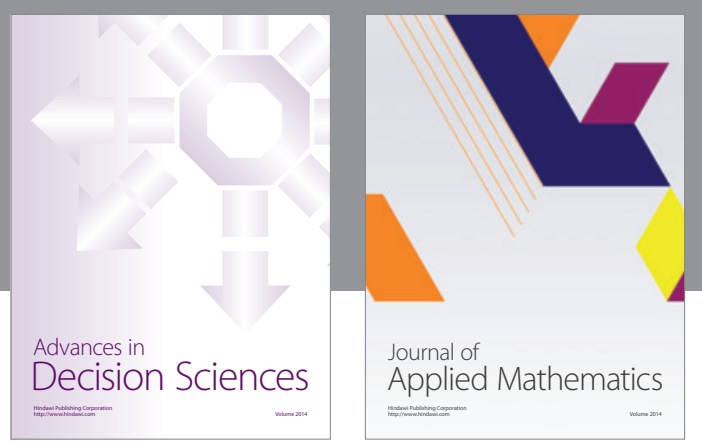

Algebra

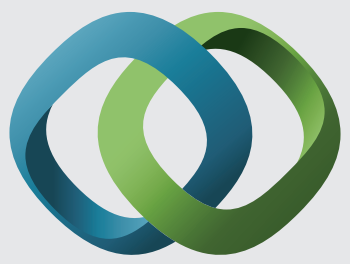

\section{Hindawi}

Submit your manuscripts at

http://www.hindawi.com
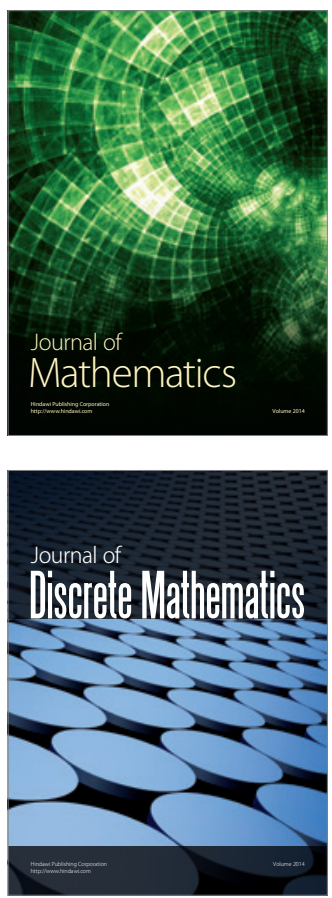

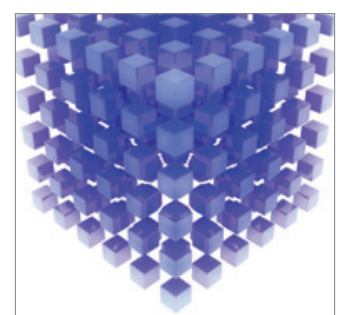

Mathematical Problems in Engineering
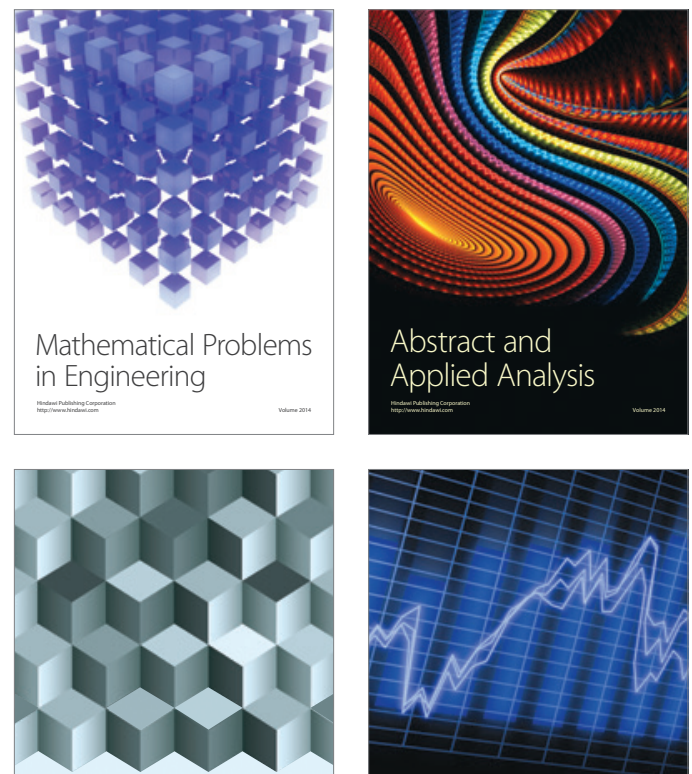

Journal of

Function Spaces

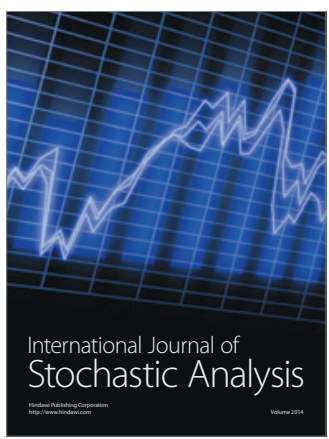

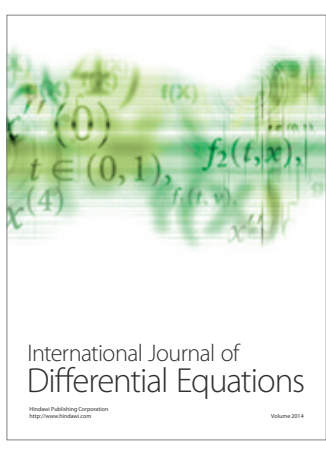
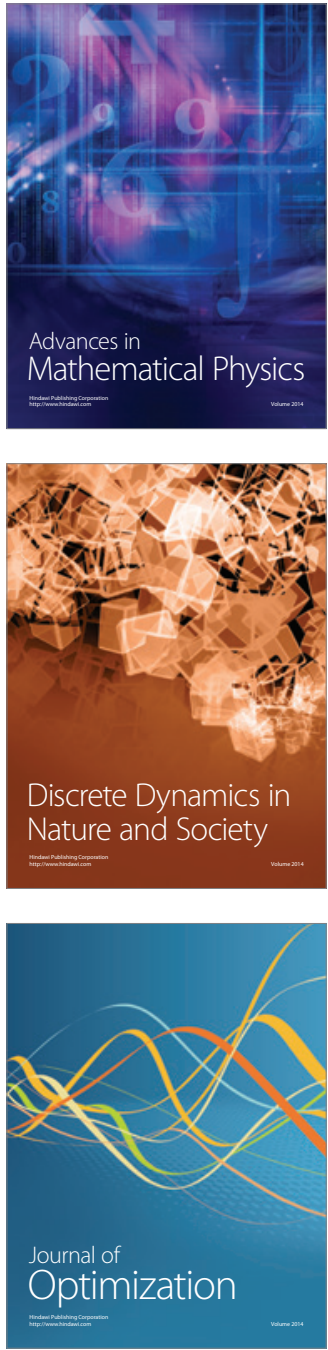